\title{
Docetaxel-based therapy with and without antiangiogenic agents as first-line chemotherapy for castration-resistant prostate cancer: A meta-analysis of nine randomized controlled trials
}

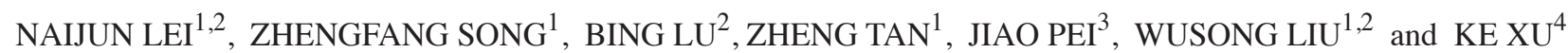 \\ ${ }^{1}$ Department of Urinary Surgery, Sichuan Cancer Hospital, Chengdu, Sichuan; ${ }^{2}$ Department of Oncology, \\ Affiliated Hospital of Guiyang Medical College and Guizhou Cancer Hospital, Guiyang, Guizhou; \\ ${ }^{3}$ Sichuan Cancer Hospital and Institute, Chengdu, Sichuan; ${ }^{4}$ Department of Oncology, \\ The First Affiliated Hospital of Chengdu Medical College, \\ Chengdu, Sichuan, P.R. China
}

Received December 12, 2013; Accepted August 8, 2014

DOI: $10.3892 / \mathrm{mco} .2014 .404$

\begin{abstract}
The aim of this study was to systematically assess the effectiveness and safety of the addition of antiangiogenic agents to docetaxel-based chemotherapy for the treatment of castration-resistant prostate cancer. Computerized electronic databases, including Embase, PubMed and The Cochrane Library were searched for randomized controlled trials (RCTs) on the comparison between docetaxel-based therapy with and without antiangiogenic agents for the treatment of prostate cancer. The search time limit was from the building of the database until July 18, 2013. Following extracting information and conducting a methodological quality evaluation for study inclusion based on inclusion and exclusion criteria, RevMan 5.2 and Stata 12.0 software were used to perform a meta-analysis and the Jadad scale was used for evaluation of study quality. A total of 9 RCTs and 4,681 patients were included in this meta-analysis. The comparison between docetaxel-based therapy with and without antiangiogenic agents revealed no statistically significant differences regarding prostate-specific antigen response rate [risk ratio $(\mathrm{RR})=0.99,95 \%$ confidence interval (CI): 0.87-1.12, $\mathrm{P}=0.84$ ], overall survival (OS) [hazard ratio $(\mathrm{HR})=0.97,95 \% \mathrm{CI}: 0.91-1.05)]$ and progression - free survival (PFS) $(\mathrm{HR}=0.99,95 \% \mathrm{CI}$ : 0.83-1.18); however, the incidence of treatment-related mortality was higher in the docetaxel-based therapy with antiangiogenic agents group
\end{abstract}

Correspondence to: Professor Zhengfang Song, Department of Urinary Surgery, Sichuan Cancer Hospital, 55 section 4, South Renmin Road, Chengdu, Sichuan 610041, P.R. China

E-mail: zfsong@sina.com

Professor Bing Lu, Department of Oncology, Affiliated Hospital of Guiyang Medical College and Guizhou Cancer Hospital, 1 Beijing Road West, Guiyang, Guizhou 550003, P.R. China.

E-mail: lbgymaaaa@sohu.com

Key words: docetaxel, antiangiogenic, castration-resistant prostate cancer, randomized controlled trial, meta-analysis
( $\mathrm{RR}=1.95,95 \% \mathrm{CI}: 1.23-3.11, \mathrm{P}=0.005)$, whereas the incidence of thrombus formation was higher in the docetaxel-based therapy without antiangiogenic agents group $(\mathrm{R} R=0.57$, 95\% CI: 0.41-0.80, $\mathrm{P}=0.001)$. In conclusion, our findings indicated that docetaxel combined with antiangiogenic agents did not increase the OS or the PFS of the patients with castration-resistant prostate cancer, whereas it may increase the risk of treatment-related mortality. However, further RCTs with larger, high-quality patient samples are required to verify these findings.

\section{Introduction}

Prostate cancer is the most common type of cancer and the leading cause of cancer-related morbidity among men in the United States of America. According to the assessment of the American Society of Clinical Oncology, there were 238,590 new cases of prostate cancer and 29,720 deaths in 2013 (1). In China, the morbidity associated with prostate cancer is significantly lower, although it has increased over the last few years. Due to the limitations in health awareness and financial resources, the majority of patients in China have middle- and advanced-stage disease at diagnosis. Although endocrine therapy may control and improve the patients' condition, the overwhelming majority are likely to develop hormone-refractory prostate cancer (HRPC) after 18-30 months of remission. Chemotherapy has been used for the treatment of HRPC for $\sim 30$ years, although the chemotherapeutic regimens were reportedly associated with severe side effects and poor curative effect. In 1996, the American Food and Drug Administration (FDA) authorized the therapeutic regimen of mitoxantrone combined with prednisone. The clinical test results revealed that, although compared to single-agent prednisone this combination treatment achieved a remission of the clinical symptoms of the patients, it failed to improve the overall survival (OS) rate (2). The results of the TAX327 phase III trial revealed that, compared to mitoxantrone, the treatment regimen combining docetaxel and prednisone improved the quality of life of the patients and prolonged their mean survival 
of 2.5 months (3). In 2004, the American FDA authorized the use of docetaxel-based therapy, which is currently the first-line standard treatment and a focus of ongoing investigation. The results of a meta-analysis of docetaxel-based therapy with or without estramustine, revealed that there were no statistically significant differences in the incidence of grade $3 / 4$ adverse events or in OS rates; however, there was a statistically significant difference in the prostate-specific antigen (PSA) response rate [odds ratio $=1.55,95 \%$ confidence interval $(\mathrm{CI}): 1.10-2.18$, $\mathrm{P}=0.012$ ] (4), suggesting that docetaxel-based therapy with estramustine may increase the PSA response rate. There are several RCTs on the combination of docetaxel with antiangiogenic agents available in the international literature; however, the results of those studies have been inconsistent. Therefore, a systematic assessment method was adopted, with the aim to evaluate the effectiveness and safety of docetaxel-based therapy with and without antiangiogenic agents for the treatment of castration-resistant prostate cancer, in order to provide the basis for clinical decision making.

\section{Materials and methods}

Study design and inclusion criteria. The study design was RCT and the record type was not limited by the blinding method. The study included patients with a definitive pathological diagnosis of prostate adenocarcinoma, exhibiting disease relapse following endocrine therapy, without underlying diseases, such as severe cardiac and/or pulmonary conditions. The patient selection was not limited by race or ethnicity.

Treatment. The study compared the combination of docetaxel-based therapy with antiangiogenic agents with docetaxel-based therapy alone. The outcome indicators were overall survival, progression-free survival (PFS), PSA response rate, grade 3/4 toxicity and treatment-related mortality.

Exclusion criteria. The studies were excluded if i) they applied inappropriate methods; ii) provided incomplete information that could not be otherwise obtained; iii) in case of duplicate publications, the one with the more precise methodology report was selected; and iv) studies with undefined outcome indicators.

Search strategy. Computerized electronic databases, including Embase, PubMed and The Cochrane Library, were searched. The search time limit was from the building of the database until July 18, 2013. The search used a method that combines subject terms [MEDLINE (Mesh), Embase (EMTREE)] and free terms. The keywords used included prostate cancer, prostatic carcinoma, carcinoma of the prostate, chemotherapy, docetaxel and randomized controlled trial, which were adjusted according to the specific database. The reference lists of the identified studies were also reviewed. If a study report was unclear or information was lacking, the author was contacted via e-mail.

Literature screening and data extraction. Data were extracted from the studies that conformed to the inclusion criteria by two independent researchers, filled into a data extraction table and cross-checked. Any differences were resolved by consulting a third researcher and the corresponding author of the study was contacted in case of missing data.

Quality assessment. An open assessment of the trials was performed using the method reported by Jadad et al (5), which assessed the trials on whether they reported i) an appropriate randomization method (score, $0-2$ ); ii) an appropriate blinding method (score, 0-2); and iii) withdrawals and dropouts (score, 0-1).

Statistical analysis. RevMan 5.2 and Stata 12.0 software were used to perform the statistical analyses. The measurement data used weighted or standard mean difference and 95\%CI, while the enumeration data used risk ratio (RR) and 95\% CI as a statistical magnitude of curative effect analysis. Heterogeneity was assessed with the Chi-square test. When there was no heterogeneity $\left(\mathrm{I}^{2}<50 \%, \mathrm{P}>0.10\right)$, a fixed-effects model was used for the analysis. In the case of heterogeneity, the possible sources were investigated. When clinical heterogeneity was detected, a random-effects model was used for analysis and subgroup or sensitivity analysis was performed based on the sources. If heterogeneity was significant, a descriptive analysis was undertaken. The indicators that could not be merged were used in the descriptive method.

\section{Results}

Characteristics of included studies. A total of 1,761 relevant articles were identified through an initial literature search. Duplicate articles were eliminated using the literature management software, EndNote X6, leaving a total of 1,450 studies. Subsequently, the titles and abstracts were read to exclude retrospective studies, non-RCTs and studies that did not conform to the intervention measures, leading to the exclusion of a further 1,435 studies that did not conform to the inclusion criteria. Of the remaining 15 relevant articles (6-20), 2 meeting abstracts $(14,16)$ and 4 studies that investigated other targeted drugs (17-20) were also excluded. The flowchart of the study selection process is shown in Fig. 1. The baseline parallel comparison of all the docetaxel-based therapy with and without antiangiogenic agents groups included in the studies is presented in Table I.

Quality assessment of the included studies. The baseline condition of the patients was reported in the 9 RCTs that were included, among which 8 RCTs were randomized and double-blind and 1 RCT was randomized and open-label. Of the 9 RCTs, 4 were phase III and 5 were phase II clinical trials.

\section{Meta analysis results.}

PSA response rate. In total, 8 studies $(6-11,13,14)$ (including 4,593 patients) compared the PSA response between the docetaxel-based therapy with and without antiangiogenic agents groups. Statistical heterogeneity was observed among the research results $\left(\mathrm{I}^{2}=79 \%, \mathrm{P}<0.0001\right)$. Subsequent to using the random-effects model to perform the meta-analysis, the result demonstrated that the differences between the two groups were not statistically significant with respect to the PSA response rate [RR=0.99, 95\%CI: 0.87-1.12)] (Fig. 2). 

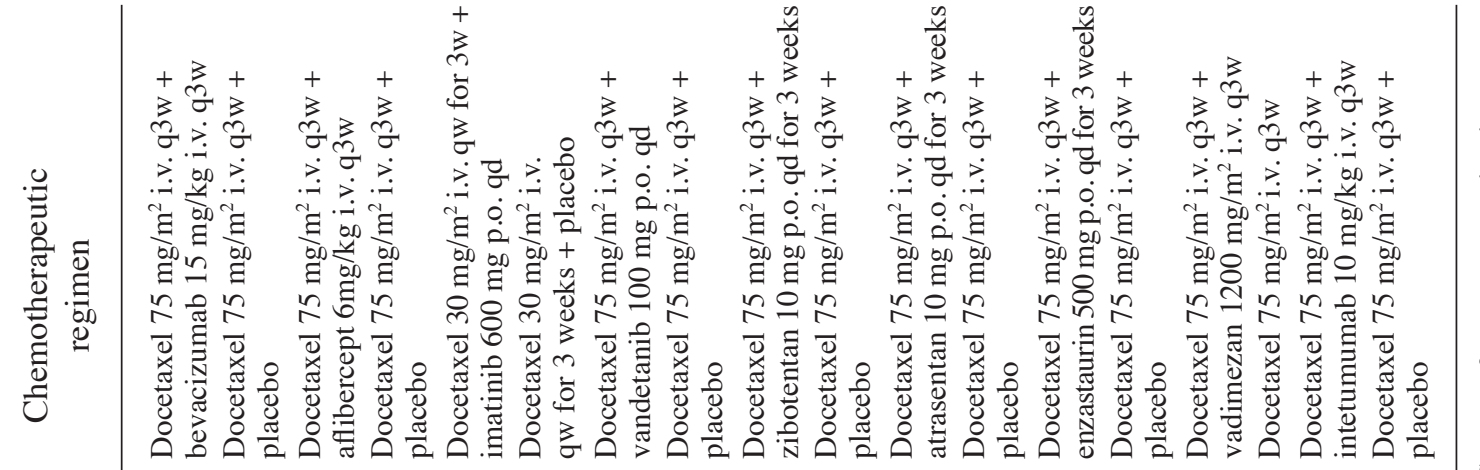

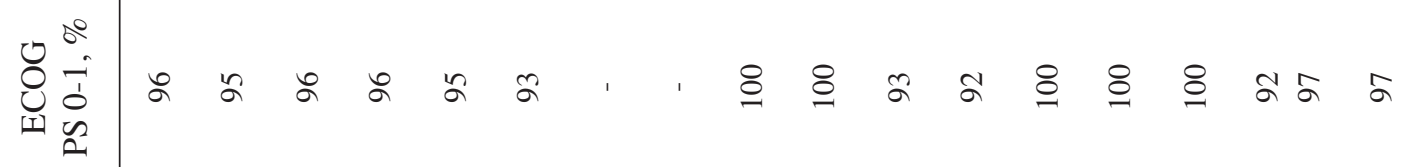

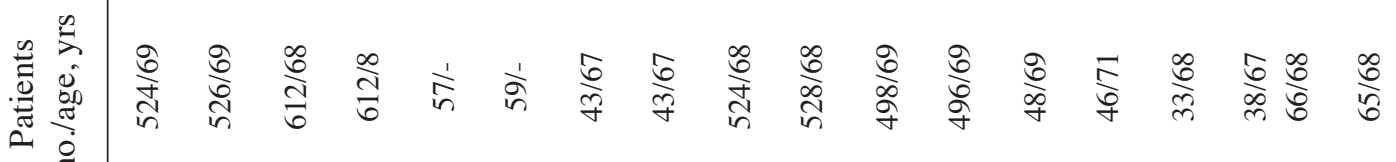

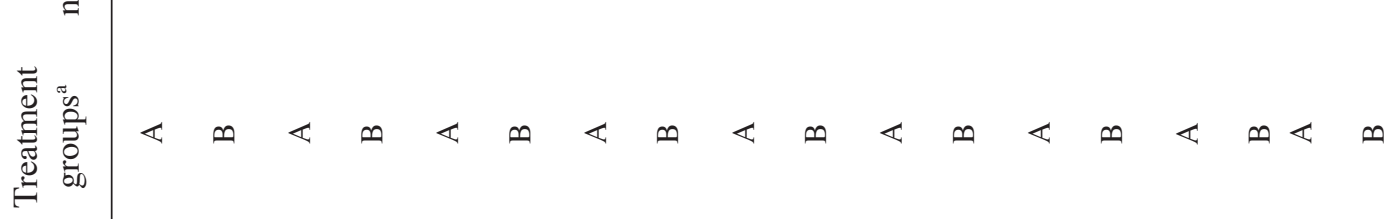
n

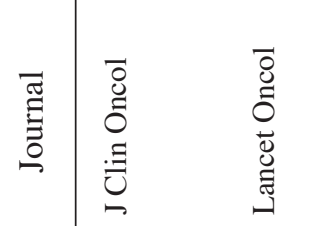

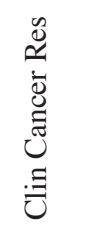

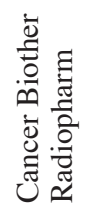

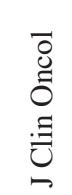

$\overrightarrow{0}$
0
0
0
0
ज్

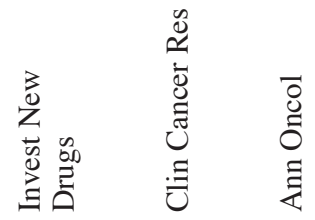

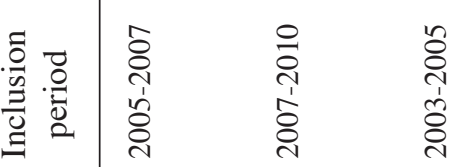

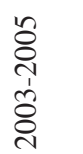

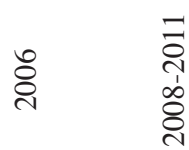

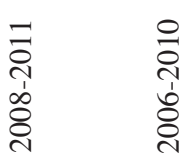

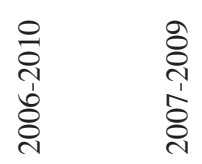

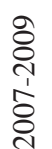

ธ
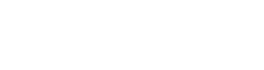
Table II. Outcome of grade 3/4 toxicity meta-analysis comparing docetaxel-based therapy with anti-angiogenesis agents vs. docetaxel-based therapy.

\begin{tabular}{|c|c|c|c|c|c|c|c|}
\hline \multirow[b]{2}{*}{ Grade 3/4 toxicity } & \multirow[b]{2}{*}{ Trials } & \multirow{2}{*}{$\begin{array}{c}\text { Combination } \\
\text { group }^{\mathrm{a}}\end{array}$} & \multirow{2}{*}{$\begin{array}{l}\text { DC alone } \\
\text { group }^{\mathrm{b}}\end{array}$} & \multicolumn{2}{|c|}{ Heterogeneity } & \multirow[b]{2}{*}{ RR $(95 \%$ CI $)$} & \multirow[b]{2}{*}{ P-value } \\
\hline & & & & $\mathrm{P}$-value & $\mathrm{I}^{2}, \%$ & & \\
\hline Neutropenia & 7 & $384 / 1,853$ & $319 / 1831$ & 0.009 & 65 & $1.16(0.91-1.47)$ & 0.230 \\
\hline Anemia & 5 & $66 / 1,181$ & $53 / 1192$ & 0.600 & 0 & $1.26(0.89-1.79)$ & 0.200 \\
\hline Diarrhea & 5 & $59 / 1,316$ & $42 / 1288$ & 0.280 & 21 & $1.36(0.93-2.00)$ & 0.120 \\
\hline Fatigue & 5 & $218 / 1,764$ & $131 / 1734$ & 0.030 & 63 & $1.49(0.99-2.25)$ & 0.060 \\
\hline Thrombosis & 4 & $50 / 1,232$ & $86 / 1203$ & 0.760 & 0 & $0.57(0.41-0.80)$ & 0.001 \\
\hline
\end{tabular}

${ }^{a}$ Docetaxel-based chemotherapy with antiangiogenic agents. ${ }^{b}$ Docetaxel-based chemotherapy without antiangiogenic agents. DC, docetaxel; RR, risk ratio; CI, confidence interval.

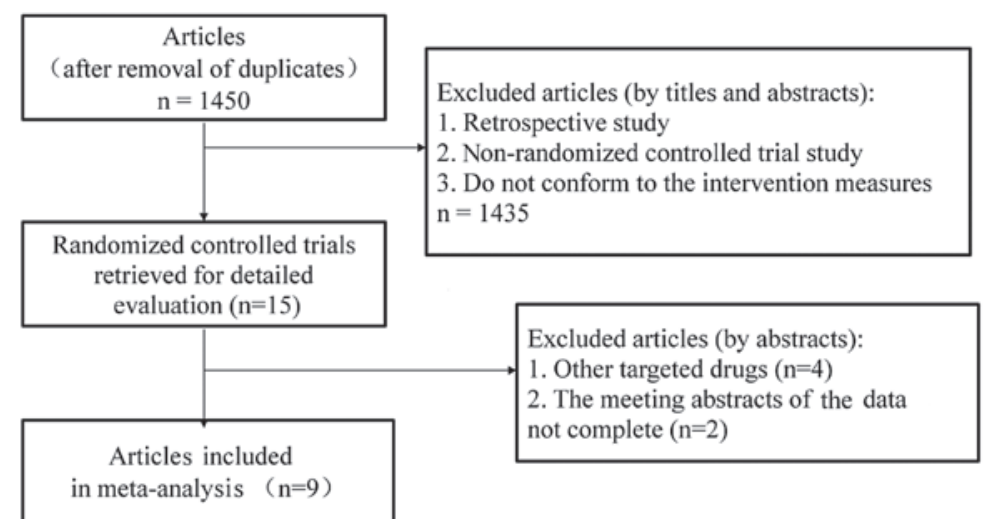

Figure 1. Studies eligible for inclusion in the meta-analysis.

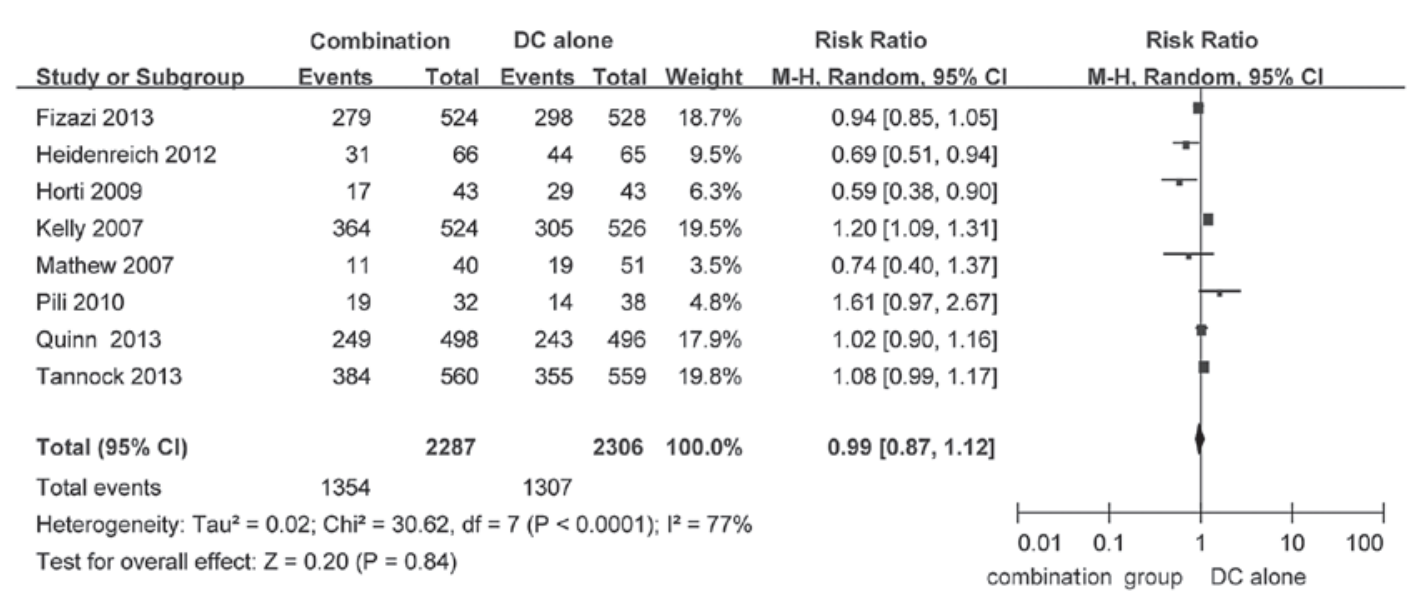

Combination group: docetaxel-based chemotherapy with antiangiogenic agents

DC alone: docetaxel-based chemotherapy without antiangiogenic agents

Figure 2. Fixed-effects model of risk ratio (95\% CI) of the PSA response rate in the docetaxel-based therapy with antiangiogenic agents group compared to the docetaxel-based therapy alone group. PSA, prostate-specific antigen; CI, confidence interval.

OS. A total of 7 studies (6-11,13,14), including 4,636 patients, reported data on the OS of the docetaxel-based therapy with and without antiangiogenic agents groups, among which 6 studies $(6,7,10,11,13,14)$, including a total of 4,521 patients, reported the results of the comparison between the two groups with respect to OS. Stata 12 software was used to perform a meta-analysis according to effect size and there was no statistical heterogeneity among the results of the included studies $\left(\mathrm{I}^{2}=0.0 \%, \mathrm{P}=0.455\right)$. Thus, the random-effects model was used for the meta-analysis and the results revealed that the 


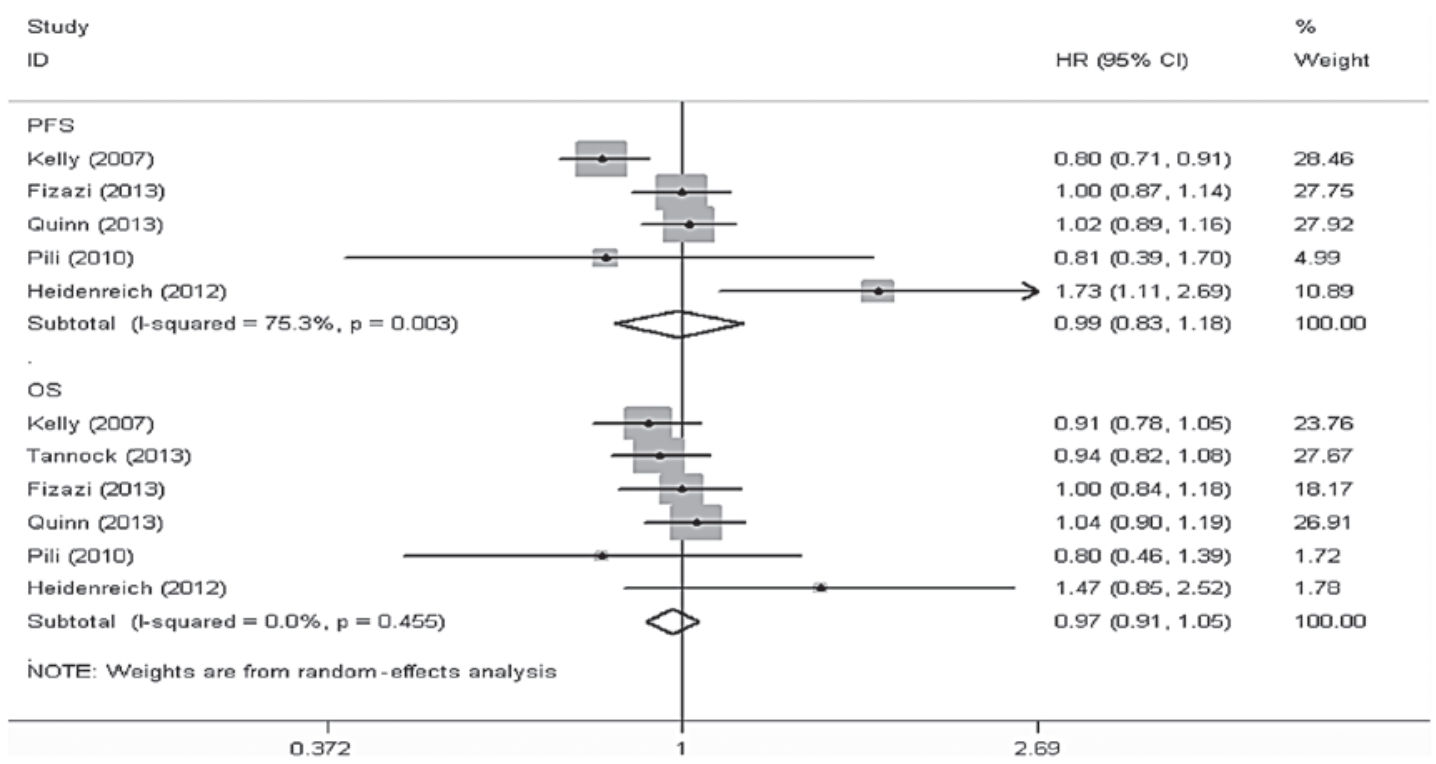

Figure 3. Fixed-effects model of hazard ratio (95\% CI) of OS and PFS associated with docetaxel-based chemotherapy with antiangiogenic agents compared to docetaxel-based chemotherapy alone. OS, overall survival; PFS, progression-free survival; HR, hazard ratio; CI, confidence interval.

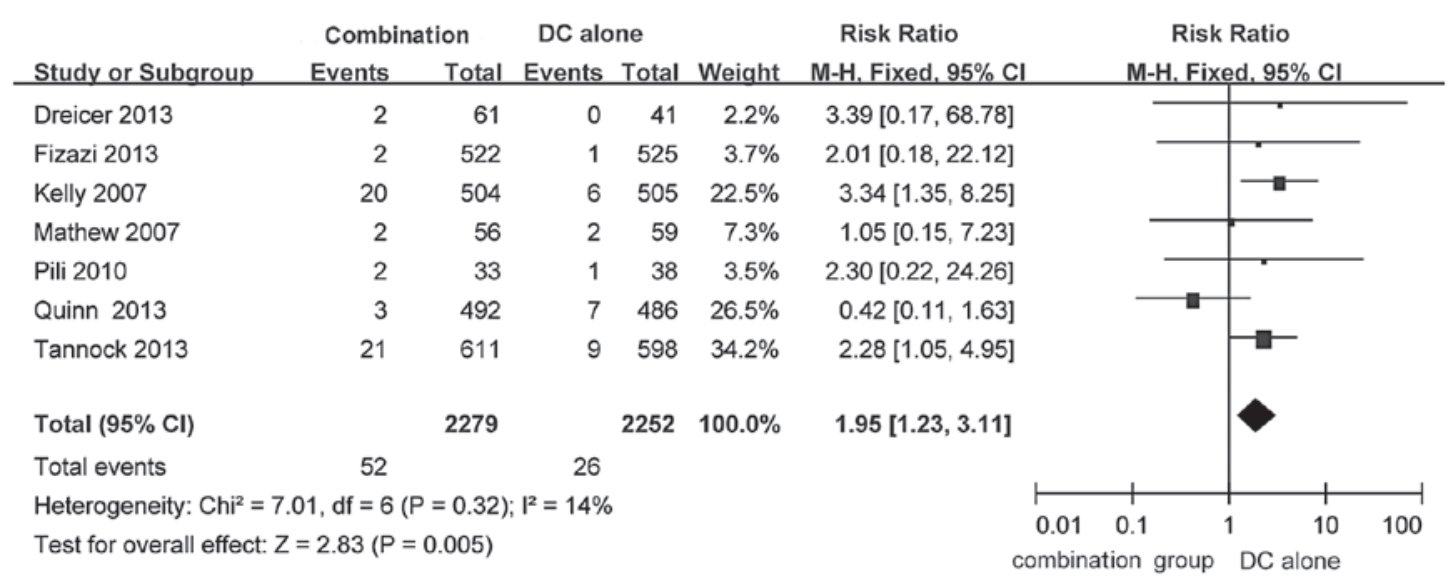

Combination group: docetaxel-based chemotherapy with antiangiogenic agents

DC alone: docetaxel-based chemotherapy without antiangiogenic agents

Figure 4. Fixed-effects model of risk ratio $(95 \% \mathrm{CI})$ of treating related mortality associated with docetaxel-based therapy with anti-angiogenesis agents compared with docetaxel-based therapy. CI, confidence intervals.

differences in OS between the two groups were not statistically significant [hazard ratio $(\mathrm{HR})=0.97,95 \% \mathrm{CI}$ : 091-1.05] (Fig. 3).

PFS. In total, 8 studies (5-8,10-13), including 4,722 patients, reported data on the PFS of the docetaxel-based therapy with and without antiangiogenic agents groups, among which 5 studies $(5,9,10,12,13)$, including a total of 3,297 patients, reported the results of the comparison between the two groups with respect to PFS. Stata 12 software was used to conduct a meta-analysis according to the effect size and there was statistical heterogeneity among the results of the included studies $\left(\mathrm{I}^{2}=75.3 \%, \mathrm{P}=0.003\right)$. Thus, the random-effects model was used for the meta-analysis and the results revealed that the differences in PFS between the two groups were not statistically significant (HR=0.99, 95\%CI: 0.83-1.18) (Fig. 3).

Adverse reactions. All the studies reported data on adverse reactions. As regards grade 3/4 neutropenia, anemia, diarrhea and fatigue, the results demonstrated that the differences between the two groups were not statistically significant (Table II), whereas with respect to thrombus incidence, there was no statistical heterogeneity among the results of the included studies $\left(\mathrm{I}^{2}=0 \%, \mathrm{P}=0.75\right)$. Thus, the fixed-effects model was used for the meta-analysis and the results revealed that the differences between the two groups were statistically significant regarding thrombus incidence $(\mathrm{RR}=0.57$, 95\% CI: $0.41-0.80, \mathrm{P}=0.001)$, with a higher incidence in the docetaxel-based therapy with antiangiogenic agents group compared to the docetaxel-based therapy alone group (Table II).

Prostate cancer-related mortality. In total, 7 studies (6-8,10-13), including 4,531 patients, reported data on prostate cancer-related mortality in the docetaxel-based therapy with and without antiangiogenic agents groups. There was no 
statistical heterogeneity among the results of the included studies $\left(\mathrm{I}^{2}=14 \%, \mathrm{P}=0.32\right)$. Thus, a fixed-effects model was used for the meta-analysis and the results demonstrated that the differences in prostate cancer-related mortality between the two groups were statistically significant $(\mathrm{RR}=1.95,95 \% \mathrm{CI}$ : $1.23-3.11$, $\mathrm{P}=0.005)$, with a higher incidence in the docetaxel-based therapy alone group compared to that in the docetaxel-based therapy with antiangiogenic agents group (Fig. 4).

\section{Discussion}

The results of the present meta-analysis revealed that, in prostate cancer, docetaxel-based therapy with antiangiogenic agents was associated with an increased mortality risk compared to docetaxel-based therapy alone. However, with respect to thrombosis $(\mathrm{RR}=0.57,95 \% \mathrm{CI}$ : 0.41-0.80, $\mathrm{P}=0.001)$, docetaxel-based therapy with antiangiogenic agents decreased the risk of thrombosis compared to docetaxel-based therapy alone. There were no significant differences between the two groups regarding OS (HR=0.97, 95\%CI: 091-1.05), PFS $(\mathrm{HR}=0.99,95 \% \mathrm{CI}: 0.83,1.18)$ and PSA response rate $(\mathrm{RR}=0.99$, 95\%CI: 0.87,1.12). Furthermore, there were no significant differences between the two groups regarding common grade $3 / 4$ adverse reactions, including neutropenia $(\mathrm{RR}=1.16$, 95\%CI: 0.91-1.47, $\mathrm{P}=0.23$, anemia $(\mathrm{RR}=1.26,95 \% \mathrm{CI}: 0.89-1.79$, $\mathrm{P}=0.20)$, diarrhea $(\mathrm{RR}=1.36,95 \% \mathrm{CI}: 0.93-2.00, \mathrm{P}=0.12)$ and fatigue $(\mathrm{RR}=1.49,95 \% \mathrm{CI}$ : $0.99-2.25, \mathrm{P}=0.06)$.

Patients with malignant tumors commonly exhibit a hyperfunctional coagulation pathway and are at high risk of thrombotic events. However, the results of the present meta-analysis with respect to thrombosis $(\mathrm{R} R=0.57$, 95\% CI: 0.41-0.80, $\mathrm{P}=0.001)$, revealed that docetaxel-based therapy with antiangiogenic agents was associated with a decreased the risk of thrombosis when treating prostate cancer. Not all studies reporting thrombosis as an adverse reaction analyzed thrombosis-related data; however, multi-targeted drugs are known to be associated with adverse reactions such as thrombosis and this conclusion is supported by numerous basic and high-quality clinical studies.

In the present meta-analysis, there were no distinct statistical differences between the docetaxel-based therapy with and without antiangiogenic agents groups with respect to PSA response rate, OS and PFS. However, as regards treatment cost, the addition of the targeted drug to the regimen clearly increases the medical expenses. For example, by combining docetaxel with bevacizumab (Avastin; Roche Group), the cost per cycle increases 5-fold compared to that of docetaxel-based therapy alone.

In the meta analysis, the dosage of docetaxel in the docetaxel-based therapy group was in line with that in the docetaxel-based therapy with antiangiogenic agents group. There was a statistically significant difference between the two groups regarding treatment-related mortality; however, whether this is associated with the drug combination or the drug dosage remains to be determined by further clinical trials including a larger patient sample. In one of the RCTs (6), docetaxel was administered at a dosage of $30 \mathrm{mg} / \mathrm{m}^{2}$ intravenously (i.v.), once per week for 3 weeks, while in other randomized trials docetaxel was administered at a dosage of $75 \mathrm{mg} / \mathrm{m}^{2}$ i.v. every 3 weeks. The results revealed that, with the dose of $30 \mathrm{mg} / \mathrm{m}^{2}$, the mean OS was 4.2 months in both treatment groups, which was significantly lower compared to the results of other randomized trials. The results of the TAX327 phase III trial (21) revealed that docetaxel at $75 \mathrm{mg} / \mathrm{m}^{2}$ i.v. every 3 weeks was superior to $30 \mathrm{mg} / \mathrm{m}^{2}$ docetaxel i.v. weekly for 5 to 6 weeks. However, in another randomized trial (22), $50 \mathrm{mg} / \mathrm{m}^{2}$ docetaxel administered i.v. on days 1 and 15 of a 4 -week cycle was superior to $75 \mathrm{mg} / \mathrm{m}^{2}$ docetaxel administered i.v. on day 1 of a 3 -week cycle, indicating that the administration schedule and dosage of docetaxel may be correlated with the results.

In the present meta-analysis, all the included studies were obtained through strict screening. According to the Jadad scale, 1 study scored 2 points and 8 studies scored $\geq 3$ points. Of the 9 RCTs, 8 were randomized double-blind and 1 RCT was randomized open-label. Four of the 9 studies were phase III and 5 were phase II clinical trials. Furthermore, a number of trials are global multicenter randomized clinical trials covering Europe, Africa and Asia. Of note, 9 antiangiogenic agents in total were included in the present meta-analysis.

\section{References}

1. Siegel R, Naishadham D and Jemal A, Cancer Statistics, 2013. CA Cancer J Clin 63: 11-30, 2013.

2. Tannock IF, Osoba D, Stockler MR, et al: Chemotherapy with mitoxantrone plus prednisone or prednisone alone for symptomatic hormone-resistant prostate cancer: a Canadian randomized trial with palliative end points. J Clin Oncol 14: 1756-1764, 1996

3. Tannock IF, Wit R, Berry WR, et al: Docetaxel plus prednisone or mitoxantrone plus prednisone for advanced prostate cancer. N Engl J Med 351: 1502-1512, 2004.

4. Qi WX, Shen Z and Yao Y: Docetaxel-based therapy with or without estramustine as first-line chemotherapy for castration-resistant prostate cancer: a meta-analysis of four randomized controlled trials. J Cancer Res Clin Oncol 137: 1785-1790,2011.

5. Jadad AR, Moore RA, Carroll D, et al: Assessing the quality of reports of randomized clinical trials: Is blinding necessary? Control Clin Trials 17: 1-12, 1996.

6. Kelly WK, Halabi S,Carducci M, et al: Randomized, double-blind, placebo-controlled phase III trial comparing docetaxel and prednisone with or without bevacizumab in men with metastatic castration-resistant prostate cancer: CALGB 90401. J Clin Oncol 30: 1534-1540, 2012.

7. Tannock IF, Fizazi K, Ivanov S, et al: Aflibercept versus placebo in combination with docetaxel and prednisone for treatment of men with metastatic castration-resistant prostate cancer (VENICE): a phase 3, double-blind randomised trial. Lancet Oncol 14: 760-768, 2013.

8. Mathew P, Thall PF, Bucana CD, et al: Platelet-derived growth factor receptor inhibition and chemotherapy for castration-resistant prostate cancer with bone metastases. Clin Cancer Res 13: 5816-5824, 2007.

9. Horti J, Widmark A, Stenzl A, et al: A randomized, double-blind, placebo-controlled phase II study of vandetanib plus docetaxel/prednisolone in patients with hormone-refractory prostate cancer. Cancer Biother Radiopharm 24: 175-180, 2009.

10. Fizazi KS, Higano CS, Nelson JB, et al: Phase III, randomized,placebo-controlled study of docetaxel in combination with zibotentan in patients with metastatic castration-resistant prostate cancer. J Clin Oncol 31: 1740-1747, 2013.

11. Quinn DI, Tangen CM, Hussain M, et al: Docetaxel and atrasentan versus docetaxel and placebo for men with advanced castration-resistant prostate cancer (SWOG S0421): a randomised phase 3 trial. Lancet Oncol 14:893-900, 2013

12. Dreicer R, Garcia J, Rini B, et al: A randomized, double-blind, placebo-controlled, phase II study with and without enzastaurin in combination with docetaxel-based chemotherapy in patients with castration-resistant metastatic prostate cancer. Invest New Drugs 31: 1044-1050, 2013. 
13. Pili R, Rosenthal MA, Mainwaring PN, et al: Phase II study on the addition of ASA404 (vadimezan; 5,6-dimethylxanthenone4-acetic acid) to docetaxel in CRMPC. Clin Cancer Res 16 2906-2914, 2010

14. Heidenreich A, Rawal SK, Szkarlat K, et al: A randomized, double-blind, multicenter, phase 2 study of a human monoclonal antibody to human $\alpha v$ integrins (intetumumab) in combination with docetaxel and prednisone for the first-line treatment of patients with metastatic castration-resistant prostate cancer. Ann Oncol 24: 329-336, 2013.

15. Heath EI, Mannuel HD, Liu G, et al: Randomized phase II trial of docetaxel (Doc) and prednisone (Pred) with or without AZD2171 (cediranib), in chemotherapy-naive, metastatic castrate-resistant prostate cancer (mCRPC) (NCI 7451). J Clin Oncol 31, (Suppl 6; abstr 38), 2013

16. Wiechno PJ, Chlosta P, Pikiel J, et al: Randomized phase II study with window-design to evaluate anti-tumor activity of the survivin antisense oligonucleotide (ASO) ly2181308 in combination with docetaxel for first-line treatment of castrate-resistant prostate cancer (CRPC). J Clin Oncol 31 (Suppl; abstr 5019), 2013.

17. Sternberg CN, Dumez H, Poppel HV, et al: Docetaxel plus oblimersen sodium (Bcl-2 antisense oligonucleotide): an EORTC multicenter, randomized phase II study in patients with castration-resistant prostate cancer. Ann Oncol 20: 1264-1269, 2009.
18. Chi KN, Hotte SJ, Yu EY, et al: Randomized phase II study of docetaxel and prednisone with or without OGX-011 in patients with metastatic castration-resistant prostate cancer. J Clin Oncol 28: 4247-4254, 2010.

19. Dahut WL, Gulley JL, Arlen PM, et al: Randomized phase II trial of docetaxel plus thalidomide in androgen-independent prostate cancer. J Clin Oncol 22: 2532-2539, 2004.

20. Sonpavde G, Matveev V, Burke JM, et al: Randomized phase II trial of docetaxel plus prednisone in combination with placebo or AT-101, an oral small molecule Bcl-2 family antagonist, as first-line therapy for metastatic castration-resistant prostate cancer. Ann Oncol 23: 1803-1808, 2012.

21. Berthold DR, Pond GR, Soban F, Wit R, Eisenberger M and Tannock IF: Docetaxel plus prednisone or mitoxantrone plus prednisone for advanced prostate cancer: updated survival in the TAX 327 study. J Clin Oncol 26: 242-245, 2008.

22. Kellokumpu-Lehtinen PL, Harmenberg U, Joensuu T, et al: 2 -weekly versus 3 -weekly docetaxel to treat castration-resistant advanced prostate cancer: a randomised, phase 3 trial. Lancet Oncol 14: 117-124, 2013. 